\title{
Prognosis in patients with coronary heart disease and breath-holding limitations: a free-breathing cardiac magnetic resonance protocol at 3.0 T
}

Keyan Wang ${ }^{1}$, Wenbo Zhang ${ }^{1}$, Shuman $\mathrm{Li}^{1}$, Xiaoming Bi ${ }^{2}$, Michaela Schmidt ${ }^{3}$, Jing An ${ }^{4}$, Jie Zheng ${ }^{5}$ and Jingliang Cheng ${ }^{1 *}$ (C)

\begin{abstract}
Background and purpose: Conventional cardiac magnetic resonance (CCMR) imaging is usually performed with breath-holding $(\mathrm{BH})$, which is adverse in patients with $\mathrm{BH}$ limitations. We explored the ability of a free-breathing CMR (fCMR) protocol to prognosticate in patients with coronary heart diseases (CHD) and limited BH ability.

Methods: Sixty-seven patients with $\mathrm{CHD}$ and limited $\mathrm{BH}$ abilities were prospectively enrolled in this study. All patients underwent comprehensive fCMR imaging at 3.0 T. The fCMR protocols included compressed sensing (CS) single-shot cine acceleration imaging, and motion-corrected (MOCO), single-shot late gadolinium enhancement (LGE) imaging. Image quality (IQ) of the cine and LGE images was evaluated based on the 5-point Likert scale. The value of fMRI in providing a prognosis in patients with CHD was assessed. Statistical methods included the T test, Mann-Whitney test, Kappa test, Kaplan-Meier curve, Log-rank test, Cox proportional hazard regression analysis, and receiver operating characteristic curves.

Results: All IQ scores of the short axis CS-cine and both the short and long axes MOCO LGE images were $\geq 3$ points. Over a median follow-up of 31 months (range 3.8-38.2), 25 major adverse cardiovascular events (MACE) occurred. In the univariate analysis, infarction size (IS), left ventricular ejection fraction (LVEF), 3D-Global peak longitudinal strain (3D-GPLS), heart failure classification were significantly associated with MACE. When the significantly univariate MACE predictors, added to the multivariate analysis, which showed IS (HR 1.02; $95 \% \mathrm{Cl} 1.00-1.05 ; p=0.048)$ and heart failure with preserved EF (HR 0.20; 95\% Cl 0.04-0.98; $p=0.048)$ correlated positively with MACE. The optimal cutoff value for LVEF, 3D-GPLS, and IS in predicting MACE was 34.2\%, - 5.7\%, and $26.1 \%$ respectively, with a sensitivity of $90.5 \%, 64 \%$, and $96.0 \%$ and specificity of $72 \%, 95.2 \%$, and $85.7 \%$ respectively.
\end{abstract}

Conclusions: The fCMR protocol can be used to make prognostic assessments in patients with CHD and BH limitations by calculating IS and LVEF.

Keywords: Coronary heart disease, Prognoses, Free-breathing, Cardiac magnetic resonance

*Correspondence: zdyfymri2008@163.com

${ }^{1}$ MRI Department, The First Affiliated Hospital of Zhengzhou University,

Zhengzhou, China

Full list of author information is available at the end of the article

\section{Background}

Coronary heart disease (CHD) is primarily caused by coronary atherosclerosis, which causes coronary artery stenosis and myocardial ischemia. These changes then lead to myocardial infarction (MI) or sudden 
death. Cardiac magnetic resonance (CMR) imaging has been used to diagnose MI for a long time [1-3]. Previous studies have demonstrated that the CMR markers of myocardial and microvascular damage add incremental prognostic information to clinical diagnoses [4-7]; however, most of these studies lack prognostic information in patients with $\mathrm{CHD}$ and $\mathrm{BH}$ impairments. An absence of accepted CMR marker cutoff values to enable optimized risk stratifications in clinical practice is also lacking. Conventional CMR (CCMR) protocols used in our center primarily include routine cine and late gadolinium enhancement (LGE) imaging. Data acquisitions are typically performed with breath-holding (BH). In clinical practice, a few of patients with CHD quit CCMR examinations because it was difficult to perform $\mathrm{BH}$ in the time needed.

Accelerated single-shot methods for cine imaging using compressed sensing (CS) acceleration [8] and LGE imaging using motion correction (MOCO) techniques are accepted for free-breathing imaging [9]. CS cine imaging has shown good consistency for assessing left ventricular function with standard cine protocols, and MOCO LGE has shown good consistency for MI detection with conventional LGE [10-13]. Although these two techniques had high consistency in cardiac functional evaluations and myocardial infarction detection compared with traditional techniques, no research has been performed on the application of these two techniques to prognosticate patients with $\mathrm{CHD}$ and $\mathrm{BH}$ limitations. This study aimed to develop a comprehensive free-breathing cardiac magnetic resonance (fCMR) protocol, including CS cine and MOCO LGE sequences, for patients with $\mathrm{CHD}$ and $\mathrm{BH}$ limitations. We also sought to explore the value of this protocol in determining prognoses in patients with $\mathrm{CHD}$ and $\mathrm{BH}$ limitations.

\section{Methods \\ Subject enrollment}

The institutional review board of our institution approved this study. We prospectively recruited adult patients scheduled for CMR examinations from February 1, 2017, to June 18, 2019. The inclusion criteria were: (1) Patients with CHD confirmed with DSA, who also had $\mathrm{BH}$ limitations and could not undergo CCMR protocols, including those with severe heart failure and coma, and other vulnerable patients; (2) A glomerular filtration rate of $\geq 30 \mathrm{~mL} / \mathrm{min}$ per $1.7 \mathrm{~m}^{2}$, and no other contraindications for CMR imaging. DSA images were analyzed for stenosis by a radiologist with greater than 10 years of experience. According to the criteria prognostically significant for CHD, CHD was diagnosed if vessel stenosis was $\geq 70 \%$ on DSA [14]. We obtained patient baseline characteristics from the electronic medical records.

\section{The CMR imaging protocol}

fCMR scans were performed on a clinical 3 T MRI scanner (MAGNETOM Skyra, Siemens Healthcare, Erlangen, Germany). The system was equipped with an 18-element body array coil and a 32-element spine array coil. The fCMR protocol primarily included: (1) CS cine imaging with balanced steady-state free precession (bSSFP) readout, featuring a two-dimensional, realtime, true fast imaging, steady-state precession, sparse data sampling and iterative reconstruction (SSIR). (2) MOCO LGE is characterized by respiratory motioncorrected, single-shot steady-state free procession, and averaged phase-sensitive inversion recovery (PSIR).

Table 1 Typical free-breathing cardiac resonance (fCMR) image acquisition parameters

\begin{tabular}{|c|c|c|c|c|}
\hline Parameters & MOCO-LGE LAX & MOCO-LGE SAX & CS-cine SAX & CS-cine LAX \\
\hline Echo time (ms) & 1.18 & 1.18 & 1.2 & 1.2 \\
\hline Repetition time (ms) & 2.8 & 2.9 & 2.8 & 2.8 \\
\hline Temporal resolution (ms) & 420 & - & 42.3 & 42.6 \\
\hline $\begin{array}{l}\text { Spatial resolution reconstructed } \\
\left(\mathrm{mm}^{3}\right)\end{array}$ & $1.4 \times 1.4 \times 6.0$ & $1.4 \times 1.4 \times 8.0$ & $1.7 \times 1.7 \times 8.0$ & $1.7 \times 1.7 \times 6.0$ \\
\hline Bandwidth (Hz/pixel) & 1085 & 1085 & 910 & 962 \\
\hline Section thickness (mm) & 6 & 8 & 8 & 6 \\
\hline Section gap (mm) & 1.2 & 1.6 & 1.6 & 1.2 \\
\hline No. of sections & 3 & $7-10$ & $7-10$ & 3 \\
\hline Flip angle (degrees) & 40 & 50 & 50 & 50 \\
\hline Breath holds (n) & 0 & 0 & 0 & 0 \\
\hline Acquisition time (s) & $37 \pm 6$ & $120 \pm 19$ & $25 \pm 5$ & $8 \pm 2$ \\
\hline Accelerate factor & 8 & 8 & 20 & 20 \\
\hline ECG mode & Prospective triggering & Prospective triggering & Adaptive triggering & Adaptive triggering \\
\hline
\end{tabular}


These parameters are shown in Table 1. CS cine images were performed in three long-axis (LAX) planes (four-, three-, and two-chamber planes) and a stack of short-axis (SAX) images covering the left ventricle (LV). MOCO LGE sequence images were acquired in the same SAX and LAX planes as the CS cine images. Intravenous gadolinium diethylenetriamine penta-acetic acid (Gd-DTPA) contrast agent was administered at a dose of $0.2 \mathrm{ml} / \mathrm{kg}$ of body weight. MOCO LGE images were acquired $10 \mathrm{~min}$ after contrast injection. The workflow is shown in Fig. 1.

\section{Image analysis} Image quality

All fCMR images were transferred to a workstation (cmr42, Circle Cardiovascular imaging, Version 5.12.1, Calgary, Alberta, Canada) for evaluation. The IQ of CS cine and MOCO LGE images were evaluated by a single reviewer with $>5$ years of experience in cardiovascular imaging diagnostic. For a randomly selected sample of 30 patients, the reproducibility of the IQ scores was evaluated by another senior observer. IQ scores were evaluated based on the 5-point Likert score ranging from 1 to $5(5=$ excellent image quality, $4=$ normal image quality, $3=$ presence of artifacts but sufficient image quality, $2=$ severe artifacts in the area of the left ventricle, and $1=$ completely nondiagnostic images) $[11,12,15]$.

\section{CS cine imaging}

Left ventricular function (LVF) and feature tracking (FT) were measured with the cmr42 software (version 5.12.1, Circle Cardiovascular Imaging, Calgary, Canada), including the LV ejection fractions (LVEF), LV end-diastolic mass (LVEDM), and 3D-Global peak longitudinal strain (3D-GPLS). The end-systolic and end-diastolic phases were detected automatically at the endocardium and epicardium contours, which were automatically delineated on the short-axis CS cine images with software and were based on the smallest and largest LV volumes over the entire cardiac cycle. Contours rendered by automated analyses were reviewed and manually adjusted whenever needed [10]. LV papillary muscles and trabeculations were included in ventricular cavity volumes [16, 17]. For FT quantifications, LV epicardial and endocardial borders were automatically or manually adjusted where needed and delineated at enddiastole of the short axis and at the 2ch, 3ch, and 4ch long-axis cine images, and propagated. The 3D-GPLS values were derived in accordance with the American Heart Association's 16-segment model, excluding the apical cap, because the apex is not beneficial in observing short-axis slices, and the measurement error is relatively large.

\section{MOCO LGE}

The presence of LGE involving the subendocardium of stenotic coronary arterial distributions was classified as ischemic LGE, otherwise, it was classified as nonischemic LGE $[3,18,19]$. The presence and patterns of LGE were visually assessed on the short- and long-axis images using the American Heart Association (AHA) 17-segment model. Segments 1, 2, 3, 7, 8, 13, 14, and 17 are supplied with blood through the left anterior descending (LAD) artery; segments 5, 6, 11, 12, and 16 are supplied through the left circumflex artery (LCX); and segments $4,9,10$, and 15 are supplied through the right coronary artery (RCA). Short-axis LGE image stacks with minimal through-plane motion were used to quantify the pixel-wise infarct size (IS) [13]. Computerassisted planimetry using the cmr42 software quantified the IS, using the full width at half-maximum technique, an accepted technique for LGE quantification [20].

\section{Follow-up}

Clinical outcomes were collected during follow-up. The primary clinical outcome of the study was the composite endpoint of major adverse cardiac events (MACE), defined as a composite of all-cause mortality, and new heart failure diagnosis. All-cause death was defined as any death during the follow-up period. Heart failure (HF) was defined as clinical syndromes with symptoms and/

fCMR workflow(about $20 \mathrm{~min}$,free breathing)

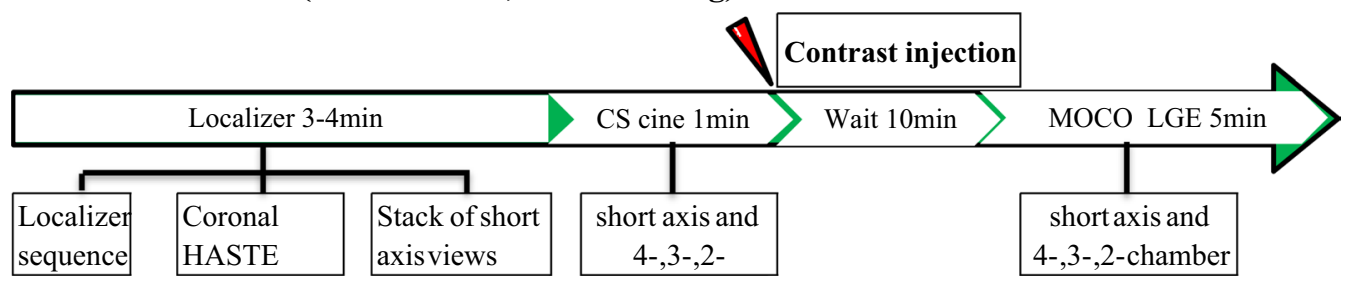

Fig. 1 The workflow of the free-breathing cardiac resonance (fCMR) protocol. HASTE half-Fourier singlc-shot turbo spin-echo, MOCO LGE motion-corrected, single-shot late gadolinium enhancement, CS compressed sensing 
or signs caused by a structural and/or functional cardiac abnormality and corroborated by elevated natriuretic peptide levels and/or objective evidence of pulmonary or systemic congestion [21]. Outcome data were collected from scheduled study follow-up visits, monthly telephone calls, and reviews of the electronic case records.

\section{Statistical analyses}

Statistical analyses were performed using the dedicated software, SPSS 20.0 (SPSS Inc., Chicago, USA) and and MedCalc10.0 (MedCalc Software, Ostend, Belgium) software. Continuous data were checked for normality using the Shapiro-Wilk test and presented as the mean \pm standard or median (interquartile range [IQR]). Qualitative variables were expressed as percentages. The Kappa statistic was used to evaluate the consistency between readers 1 and 2 for the IQ assessments. KaplanMeier survival analysis was used to analyze qualitative variables associated with MACE, reporting $X^{2}$. Cox proportional hazards regression analysis was used to analyze continuous variables associated with MACE, reporting hazard ratios (HRs) and 95\% confdence intervals (CIs). In our study, because the sample was small, variables showing values of $p<0.01$ on univariate analysis were entered into multivariate Cox proportional hazard regression analysis. Cumulative survival rate and rate of MACE were estimated using a Kaplan-Meier curve evaluated by the log-rank test. Receiver operating characteristics (ROC) curves, used to derive the optimal cutoff points of LVEF, 3D-GPLS, and IS, forecasted the MACE from the non-MACE group. Areas under the curve (AUC), sensitivities, and specificities were respectively calculated. Probability values were 2-sided, and values of $p<0.05$ were considered significant.

\section{Results}

\section{Patient characteristics}

Over two years, from February 1, 2017, to June 18, 2019, a total of 9256 patients underwent CMR imaging in our department; $67(0.72 \%)$ patients were diagnosed with CHD by DSA and unable to meet the demands of multiple $\mathrm{BH}$ sessions and long examination times. Of these 67 enrolled patients, 35 had single coronary artery stenosis, 17 had two coronary artery stenoses, 15 cases had three coronary artery stenoses. 51 cases had LAD stenoses, and the mean percentage of patients with stenosis was 90\% (85-100\%). 33 patients had LCX stenoses, and the mean percentage of patients with stenosis was $80 \%$ (75-90\%). 30 patients had RCA stenoses, and the mean percentage of patients with stenosis was $95 \%(80-100 \%)$. The demographic characteristics of the cohort are shown in Table 2.
Table 2 Demograohic characteristics of the study cohort

\begin{tabular}{|c|c|}
\hline Demographic characteristics & CHD $(n=67)$ \\
\hline Age, years & $59 \pm 12$ \\
\hline Male, n (\%) & $49(73.1)$ \\
\hline Body mass index, $\mathrm{kg} / \mathrm{m}^{2}$ & $1.74 \pm 0.22$ \\
\hline Resting heart rate > 100 bpm, n (\%) & $0(0)$ \\
\hline History of hypertension, n (\%) & $35(52.2)$ \\
\hline History of diabetes, n (\%) & $21(31.3)$ \\
\hline History of hypercholesterolemia, n (\%) & $18(26.9)$ \\
\hline Heavy tobacco use, n (\%) & $25(37.3)$ \\
\hline Family history of CHD, n (\%) & $14(20.9)$ \\
\hline Percutaneous coronary intervention, $\mathrm{n}(\%)$ & $42(62.7)$ \\
\hline Cardiac bypass surgery, n (\%) & $2(3.2)$ \\
\hline$\beta$-Blocker, n (\%) & $53(79.1)$ \\
\hline Calcium channel blocker, n (\%) & $49(73.1)$ \\
\hline ACE inhibitor, n (\%) & $25(32.5)$ \\
\hline Aspirin, $n(\%)$ & $67(100)$ \\
\hline Statin, n (\%) & $60(89.6)$ \\
\hline Nonsinus rhythm, n (\%) & $0(0)$ \\
\hline Left bundle-branch block, n (\%) & $9(13.4)$ \\
\hline Right bundle-branch block, n (\%) & $3(4.5)$ \\
\hline Troponin, ng/ml & 0.09 (0.01 to 1.06$)$ \\
\hline $\mathrm{BNP}, \mathrm{pg} / \mathrm{ml}$ & 726 (336 to 1550$)$ \\
\hline Left ventricular ejection fraction, \% & $43.5 \pm 19.0$ \\
\hline Left ventricular end-diastolic volume, $\mathrm{ml}$ & 167.7 (124 to 223.9) \\
\hline Left ventricular end-systolic volume, ml & 87.5(53.3 to 152.9$)$ \\
\hline Left ventricular stroke volume, ml & $69.9 \pm 27.8$ \\
\hline Left ventricular end-diastolic mass, g & $117.7 \pm 35.3$ \\
\hline 3D-Global peak longitudinal strain, $\%$ & $-8.5(-5.4$ to -11.2$)$ \\
\hline Infarction size, $\%$ & 22.4 (9 to 40$)$ \\
\hline Heart failure with preserved EF, n (\%) & $20(29.9)$ \\
\hline Heart failure with mid-range $\mathrm{EF}, \mathrm{n}(\%)$ & $22(32.8)$ \\
\hline Heart failure with reduced EF, n (\%) & $25(37.3)$ \\
\hline Left anterior descending stenosis, \% & $90(85-100)$ \\
\hline Left circumflex artery stenosis, \% & $80(75-90)$ \\
\hline Right coronary artery stenosis, $\%$ & $95(80-100)$ \\
\hline
\end{tabular}

\section{Image quality}

The SAX CS cine image quality scores were 3 (50.5\%), 4 (39.6\%), and 5 (9.9\%) points, and the median IQR was 3 (3-4) points. The LAX CS cine image quality scores were 2 (18.2\%), 3 (46.8\%), 4 (33.2\%), and $5(1.8 \%)$ points, and the median IQR was $3(3-4)$ points. The SAX MOCO LGE image quality scores were 3 (10.8\%), 4 (15.3\%), and $5(73.9 \%)$ points, and the median IQR was $5(4-5)$ points.The LAX MOCO LGE image quality scores were 3 (12.6\%), 4 (55.9\%), and 5 (31.5\%) points, and the median IQR was 4 (4-5) points. Readers 1 and 2 had good concordance in their decisions on the IQ assessments; SAX CS cine [IQ, kappa:0.80, 95\% CI (0.70-0.92)], 

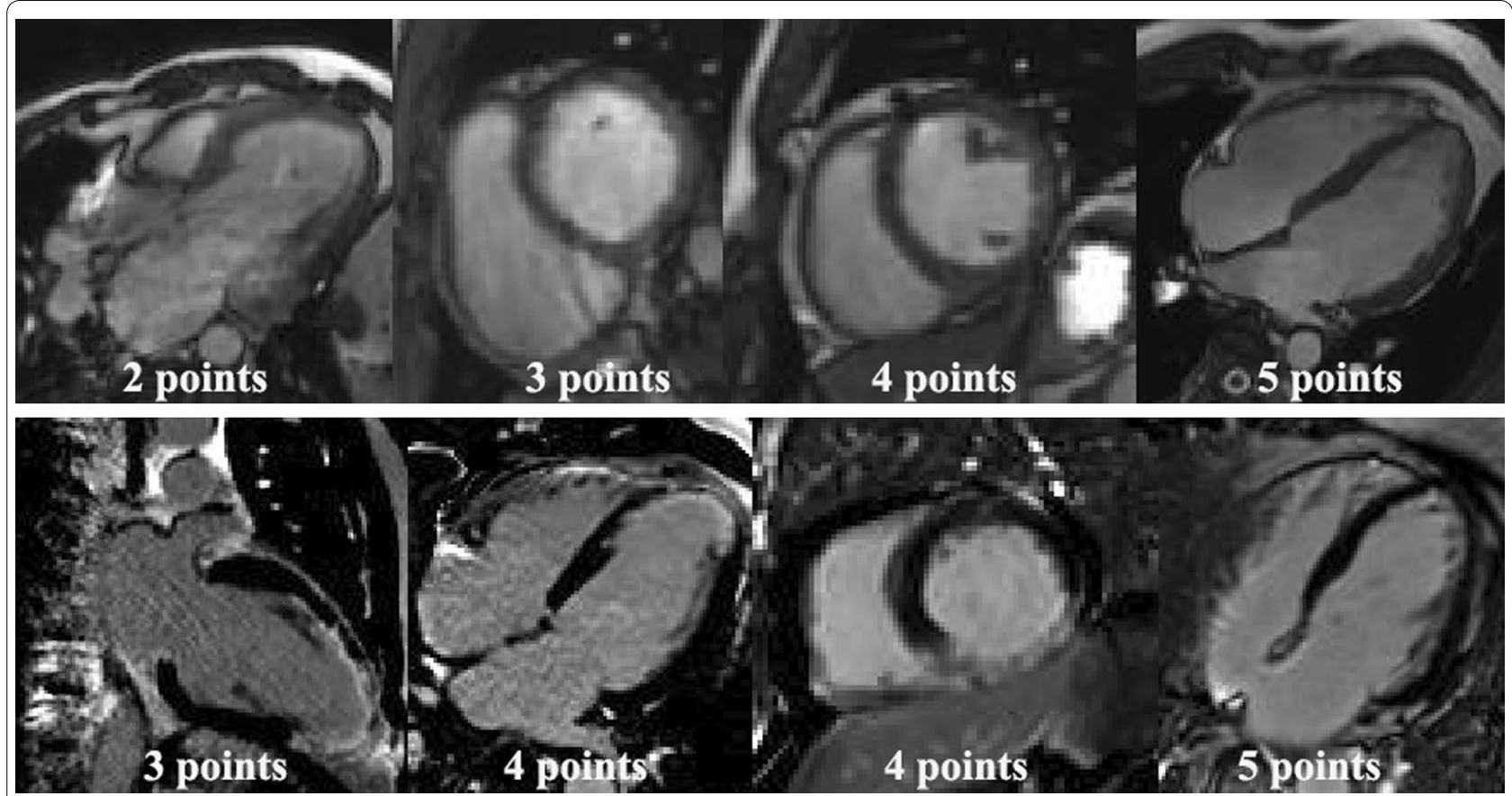

Fig. 2 Typical images display with different scores ranging from 2 to 5.2 points: sever artifact; 3 points: presence of artifacts but acceptable; 4 points: good; 5 points: excellent. Our results lack images with an image quality score of 1 point

LAX CS cine [IQ, kappa:0.72, 95\%CI (0.67-0.90)], SAX MOCO LGE [IQ, kappa:0.94, 95\%CI (0.89-1.00)], and LAX MOCO LGE [IQ, kappa:0.85, 95\%CI (0.78-0.92)]. Typical cases of IQ points is shown in Fig. 2.

\section{fCMR parameters}

After reviewing fCMR findings in the study, MOCO LGE detected 62 cases with positive ischemic LGE, 15 cases had non-ischemic LGE. Representative cases are shown in Fig. 3. The mean fCMR parameters value of 67 subjects were as follow: infarction size $22.9 \%$ (9 to $40 \%$ ), LVEF $43.5 \% \pm 19.0 \%$, LVEDV $167.7 \%$ (124 to $223.9 \%$ ), LVESV $87.5 \%$ (53.3 to $152.9 \%$ ), LVSV $69.9 \% \pm 27.8 \%$, LVEDM $117.7 \mathrm{~g} \pm 35.3 \mathrm{~g}, 3 \mathrm{D}-\mathrm{GPLS}-8.5 \%$ ( -5.4 to $-11.2 \%)$. 67 patients were divided into 3 groups according 2021 ESC Guidelines for the diagnosis of heart failure [21]: 20 patients were diagnosed as heart failure with preserved EF (HFpEF; defined as $\mathrm{EF} \geq 50 \%$ ), 22 patients were diagnosed as heart failure with mid-range EF (HFmrEF; an $\mathrm{EF}$ of $40-49 \%), 25$ patients were diagnosed as heart failure with reduced $\mathrm{EF}$ (HFrEF; defined as $\mathrm{EF}<40 \%$ ).

\section{Survival analyses@@@}

All the enrolled patients completed follow-up over a median of 31 months ( 3.8 to 38.2 months), 25 composite events $(37.3 \%, 10$ deaths, and 15 hospitalizations due to recurrence of heart failure) were observed.
Cox proportional hazards regression analysis was performed to investigate associations between baseline characteristics, fCMR parameters and MACE (Table 3). In the univariate analysis, IS (hazard ratio [HR] 1.05; 95\% confidence interval [CI] 1.03-1.07; $p<0.001$ ), LVEF (HR 0.94; 95\% CI 0.92-0.94; $p<0.001$ ), 3D-GPLS (HR 1.15; 95\% CI 1.09-1.29; $p<0.001)$, LVEDM, heart failure, age, diabetes mellitus, hypertension, ACE inhibitor use were significantly associated with MACE. LVEDV, LVESV, and LVSV were used to calculate LVEF. Therefore, LVEDV, LVESV, and LVSV were not incorporated into the univariate and multivariate analysis. According to the rule of thumb for multivariate analyses, 25 incidents were recorded during the follow-up, maximum 4 variables should be included in the multivariate analysis. The purpose of the study was to evaluate the value of fCMR parameters in the prognosis of patients with CHD and $\mathrm{BH}$ limitation, three $\mathrm{FCMR}$ parameters including IS, LVEF and 3D-GPLS, and HF classification were chose in the multivariate analysis. Multivariate analysis showed IS (HR 1.02; 95\% CI 1.00-1.05; $p=0.048$ ) and HFpEF (HR 0.20 ; $95 \%$ CI $0.04-0.98 ; p=0.048)$ correlated positively with MACE.

All three fCMR parameters had reasonable accuracy in predicting MACE. The optimal cutoff value for LVEF in predicting MACE was $34.2 \%$, with a sensitivity of $90.5 \%$ and specificity of $72 \%$. The optimal cutoff value 

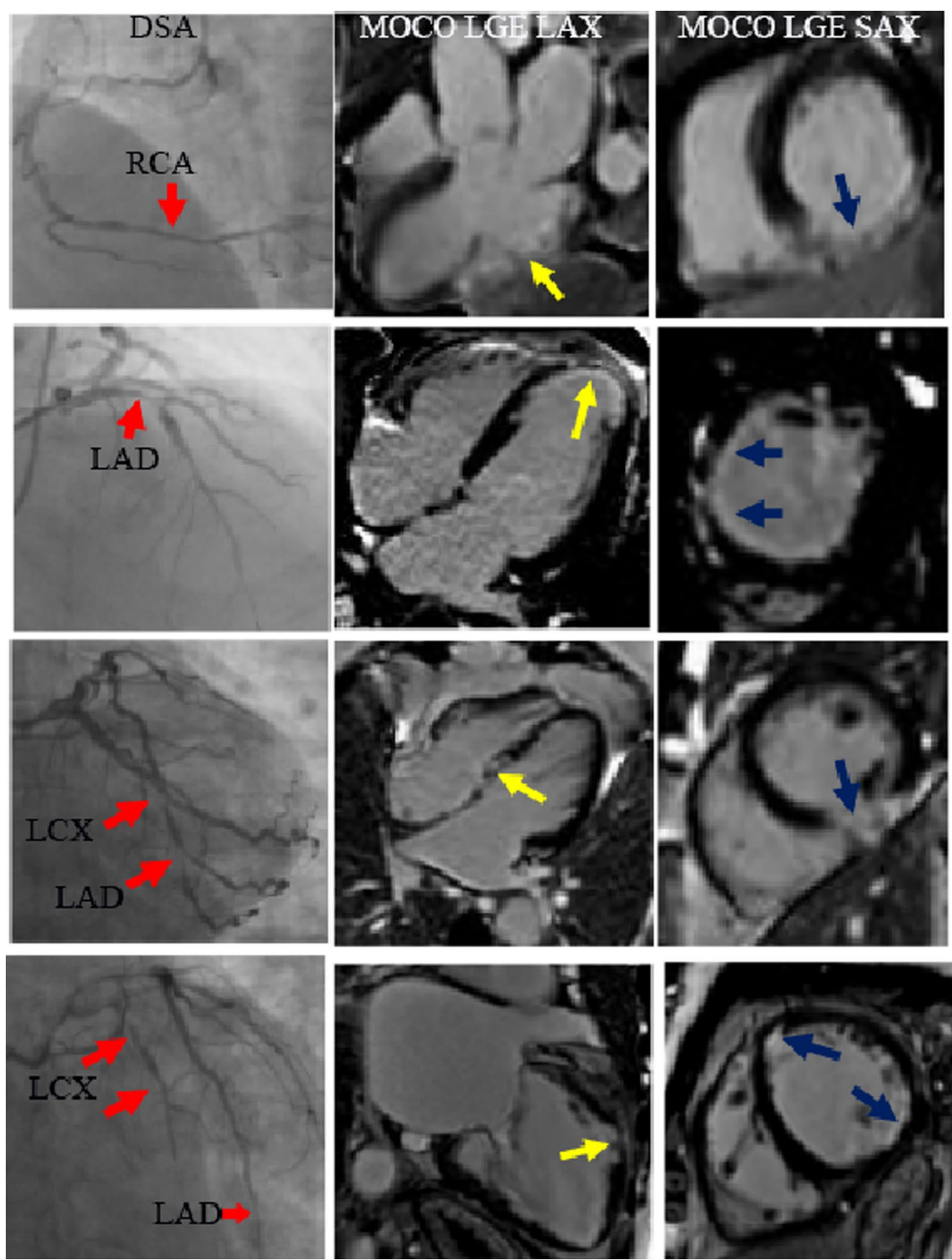

Fig. 3 Representative images of patients with coronary heart disease, including SAX and LAX MOCO LGE, and DSA. LAD left anterior descending artery, LCX left circumflex artery, RCA right coronary artery, SAX short-axis, LAX long-axis, MOCO LGE motion-corrected late gadolinium enhancement, DSA digital subtraction angiography

for 3D-GPLS in predicting MACE was $-5.7 \%$, with a sensitivity of $64 \%$ and specificity of $95.2 \%$. The optimal cutoff value for IS in predicting MACE was $26.1 \%$, with a sensitivity of $96.0 \%$ and specificity of $85.7 \%$. The AUC for LVEF, 3D-GPLS, IS was 0.854, 0.786, and 0.703, respectively (all, $p<0.05$ ).

Kaplan-Meier analyses showed a significant and progressive decrease in survival rates in the patients with HFpEF group, followed by the HFmrEF group, 
Table 3 Cox proportional hazards regression analysis for MACE

\begin{tabular}{|c|c|c|c|}
\hline Univariate predictors & $\mathrm{HR} / \mathrm{X}^{2}$ & $95.0 \% \mathrm{Cl}$ & $P$-value \\
\hline IS (per 1\% increase) & 1.05 & $1.03-1.07$ & $<0.001$ \\
\hline LVEF (per 1\% increase) & 0.94 & $0.92-0.96$ & $<0.001$ \\
\hline LVEDM (per $1 \mathrm{~g}$ increase) & 1.01 & $1.00-1.02$ & 0.032 \\
\hline 3D-GPLS (per 1\% decrease) & 1.15 & $1.09-1.29$ & $<0.001$ \\
\hline Heart failure classification & 36.2 & & $<0.001$ \\
\hline Male gender & 0.01 & & 0.929 \\
\hline Age (per 1 year increase) & 1.06 & $1.03-1.09$ & 0.030 \\
\hline Diabetes mellitus & 4.24 & & 0.039 \\
\hline Hypertension & 8.13 & & 0.004 \\
\hline Hyperhomocysteinemia & 0.08 & & 0.783 \\
\hline Smoker & 0.00 & & 0.989 \\
\hline $\mathrm{PCl}$ & 3.26 & & 0.071 \\
\hline Aspirin use & 3.14 & & 0.076 \\
\hline ACE inhibitor use & 4.43 & & 0.035 \\
\hline Beta-blocker use & 3.74 & & 0.053 \\
\hline Calcium channel blocker use & 1.41 & & 0.235 \\
\hline Statin use & 0.50 & & 0.478 \\
\hline Multivariate predictors & HR & $95.0 \% \mathrm{Cl}$ & $P$ value \\
\hline IS (per 1\% increase) & 1.02 & $1.00-1.05$ & 0.048 \\
\hline LVEF (per 1\% increase) & 1.03 & $0.96-1.09$ & 0.428 \\
\hline 3D-GPLS (per 1\% decrease) & 1.15 & $0.92-1.44$ & 0.199 \\
\hline HFpEF & 0.20 & $0.04-0.98$ & 0.048 \\
\hline
\end{tabular}

IS infarction size, LVEF left ventricular ejection fraction, LVEDV left ventricular end-diastolic volume, LVESV left ventricular end-systolic volume, LVSV left ventricular systolic volume, LVEDM left ventricular end-systolic mass, 3D-GPLS three-dimensional global peak longituditial strain, $P C I$ percutaneous coronary intervention, $H F$ hear failure, HFpEF heart failure with preserved $\mathrm{EF}$

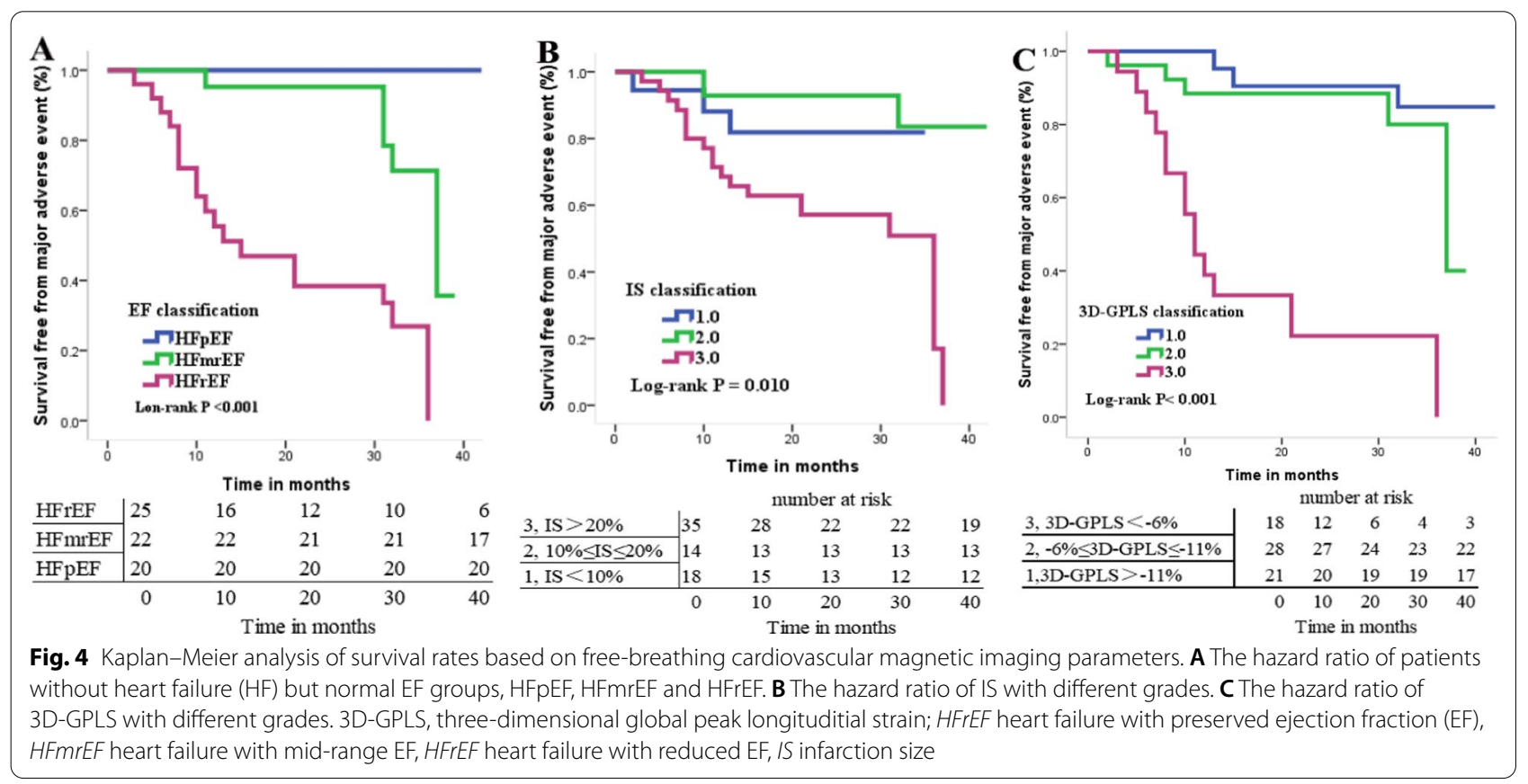


and then by the HFrEF group $(p<0.001)$ (Fig. 4A). The Kaplan-Meier analysis showed a significantly lower survival rate in the IS $>20 \%$ group than in the IS $\leq 20 \%$ group $(p=0.010)$ (Fig. 4B), and a significantly lower survival rate in the $3 \mathrm{D}-\mathrm{GPLS}<-6 \%$ group than in the $3 \mathrm{D}-\mathrm{GPLS} \geq-6 \%$ group $(p<0.001)$ (Fig. $4 \mathrm{C}$ ).

\section{Discussion}

The data in this study was derived from patients with $\mathrm{CHD}$ and $\mathrm{BH}$ limitation who could not perform CCMR examinations and used fCMR as an alternative examination. The fCMR protocol obtained robust images and could be used to perform prognostic assessments of patients with $\mathrm{CHD}$ and $\mathrm{BH}$ limitation. This protocol can be used as an alternative imaging technique for patients with breath-holding restrictions.

In the study, CCMR imaging was not performed on patients who could not hold their breath for an extended amount of time since the CCMR acquisitions required multiple breath-holds to avoid respiratory image artifacts $[22,23]$. Also, to achieve sufficiently high spatial and/or temporal resolutions during CCMR imaging, segmented k-space data are acquired over multiple heartbeats resulting in segmented data acquisitions that are prone to motion artifacts, leading to suboptimal image quality. Motion artifacts on CCMR images of patients with $\mathrm{BH}$ limitations are widely recognized, meaning that CCMR imaging in these patients has mostly been abandoned. Single-shot readout protocols effectively eliminate breathing motion artifacts in both CS cine [24-26] and MOCO LGE images [27]. Moreover, MOCO LGE is characterized as a protocol that provides motion correction and has allowed the fCMR protocol to obtain robust images under free-breathing, which has been confirmed by our study.

Generally, the validation of novel techniques is often performed through comparative studies with reference techniques considered as the gold standard. For LVF quantification, standard segmented $\mathrm{BH}$ cine imaging is the most accurate and reproducible imaging technique $[10,12]$. However, this method cannot be performed in our patients with $\mathrm{BH}$ limitations. Therefore, in this study, we showed that almost all CS cine imaging yielded robust SAX images; the high IQ scores of the CS cine images translated into high reliability for measuring left ventricular function and strain. A few LAX CS cine images had poor IQ scores, similar to what has been previously published [28], which could have been due to flow-related artifacts occurring in the phase-encoding direction during systole since the temporal domain sparsity might be limited in the anatomic regions where there is very high flow [29]. LGE have been widely used to detect subendocardial infarcts in patients with CHD, determine the extent of $\mathrm{MI}$, and provide diagnostic information for the treatment and prognosis of patients with CHD [4, 30]. Previous studies have reported no differences in MI detection between MOCO LGE and conventional LGE $[13,28]$. Although we did not obtain conventional LGE imaging, all MOCO LGE imaging yielded diagnostic images. In our study, fifteen patients with CHD confirmed by DSA were diagnosed as non-ischemic LGE on MOCO LGE imaging. This negative finding after MOCO LGE imaging could be explained by incomplete cardiac coverage, missed small subendocardial LGE due to poor contrast with the blood pool [31], or good compensation and no MI occurrence in the patients with CHD.

In this study, $37.3 \%$ of patients experienced MACE during a median follow-up of 31 months. This incidence of MACE is higher than in previous studies [4-7] and is likely because all patients in the study had $\mathrm{BH}$ impairments, and most were vulnerable. Our study was first to show that HFpEF, and IS derived from MOCO LGE, had independent values in predicting MACE in patients with CHD and BH limitation. LVEF remains the primary parameter for HF characterization and the primary inclusion criterion for clinical trials of HF [32]. The LVEF $\leq 35 \%$ derived from standard segmented $\mathrm{BH}$ cine imaging has been recognized as a strong predictor of adverse outcomes [5, 7]. In our study, the optimal cutoff value for LVEF, derived from CS cine, in predicting MACE was $34.2 \%$, with a high sensitivity but low specificity. Considering the multiple mechanisms involved in MACE, it seems unlikely that LVEF will provide adequate prognosis information for all patients [5]. Comprehensive CMR stratification tools, including 3D-GPLS and IS, could improve risk stratification significantly. 3D-GPLS is, perhaps, a more sensitive measure of myocardial contractile function than LVEF [4]. In our study, the optimal cutoff value for 3D-GPLS and IS in predicting MACE was - 5.65\% and $26.1 \%$, respectively. 3D-GPLS had high specificity but low sensitivity, whereas IS had both high sensitivity and specificity. LVEF, 3D-GPLS, and IS have individual advantages in providing prognoses and can complement one another. The benefit of the fCMR protocol is not limited to a patient's BH ability, broadening CMR imaging applications and allowing the realization of imaging goals in patients with $\mathrm{BH}$ limitations. The fCMR protocol has been successfully applied to the prognostic evaluations of patients with CHD, making up for the deficiency of CCMR imaging while allowing patients the opportunity to obtain CMR images. Therefore, cardiologists can understand the occurrence, development, and prognosis of patients with $\mathrm{CHD}$ and $\mathrm{BH}$ limitation, and further to give accurate and individualized treatments. 
There were some limitations to this study. First, it was a single-center study and, therefore, reflects the use of clinical protocols at our institution. Another major limitation is the lack of CCMR imaging, and due to practical reasons, fCMR imaging was systematically performed when CCMR methods could not be implemented, which could have selected for a group that benefited most from fCMR imaging. The sample size was also small, and the follow-up time was short. Future studies should be performed with larger sample sizes and longer follow-up times. Moreover, patients with CHD should have received optimal medical treatments according to the hospital guidelines, such as receiving the combination medical regimens, including sacubitril/ valsartan. In our study, the absence of assessing the use of these medications is a limitation for risk assessments. Finally, the fCMR protocol did not include advanced MR sequences, such as mapping, perfusion, and blood flow. We did not evaluate the incremental benefit of T2-weighted STIR imaging for myocardial edema because an optimized sequence in currently not available.

\section{Conclusions}

For patients who were unable to undergo CCMR imaging, the fCMR protocols obtained robust images that could be used to evaluate cardiac function and detect myocardial infarction, and could be used to perform prognostic assessments. This protocol is promising as an alternative technique to CCMR and could replace CCMR in the future.

\section{Abbreviations \\ LGE: Late gadolinium enhancement; IS: Infarction size; LV: Left ventricle; LVEDV: Left ventricular end-diastolic volume; LVEF: Left ventricular ejection fraction; LVESV: Left ventricular end-systolic volume; LVSV: LV stroke volumes; CMR: Cardiac magnetic resonance; ECG: Electrocardiogram; LVF: Left ventricular function; FT: Feature tracking; 3D-GPLS: 3D-Global peak longitudinal strain; CHD: Coronary heart diseases; BH: Breath-holding; fCMR: Free-breathing CMR; MOCO: Motion-corrected; CS: Compressed sensing; IQ: Image quality; MACE: Major adverse cardiovascular events; MI: Myocardial infarction; AHA: American Heart Association; LAD: Left anterior descending; LCX: Left circumflex artery; RCA: Right coronary artery; ROC: Receiver operating characteristics; AUC : Area under the curve; LAX: Long-axis; SAX: Short axis; Gd-DTPA: Gadolinium diethylenetriamine pentaacetic acid; bSSFP: Balanced steady-state free procession; SSIR: Sparse data sampling and iterative reconstruction.}

\section{Acknowledgements}

We thank all the technicians in the magnetic resonance department of the First Affiliated Hospital of Zhengzhou University.

\footnotetext{
Authors' contributions

KYW (MD) conceived the study, performed the statistical analyses, and drafted the manuscript. Both WBZ (MD) and SML (MD) quantitatively measured cardiac function and infarction size, and analyzed image quality. XMB (PhD) participated in the sequence development, assisted in the interpretation of the results, and helped to revise the manuscript. MS (PhD) contributed to the sequence development and scanner implementation and helped to revise the manuscript. JA (MD) participated in the study design and scanner implementation and helped to revise the manuscript. JZ (PhD) helped to
}

revise the manuscript. JLC (MD) assisted in the interpretation of the results and helped to revise the manuscript. All authors read and approved the final manuscript.

\section{Funding}

Not applicable.

\section{Availability of data and materials}

The datasets generated for this study are available on reasonable request to the corresponding author.

\section{Declarations}

\section{Ethics approval and consent to participate}

Approval was obtained from the First Affiliated Hospital of Zhengzhou University ethics committee. All subjects/ legal guardians gave written consent and assent as appropriate.

\section{Consent to publish}

The local ethics committee approved the use of images and content from this study. Consent and assent were signed for all subjects prior to publication.

\section{Competing interests}

The authors declare that they have no competing interests.

\section{Author details}

${ }^{1}$ MRI Department, The First Affiliated Hospital of Zhengzhou University, Zhengzhou, China. ${ }^{2}$ Siemens Medical Solulations USA, Inc., Los Angeles, USA. ${ }^{3}$ Siemens Healthcare GmbH, Erlangen, Germany. ${ }^{4}$ Siemens Shenzhen Magnetic Resonance Ltd, Shenzhen, China. ${ }^{5}$ Mallinckrodt Institute of Radiology, Washington University in St. Louis, St. Louis, Missouri, USA.

Received: 8 August 2021 Accepted: 24 November 2021

Published online: 07 December 2021

\section{References}

1. Kim RJ, Albert TSE, Wible JH, et al. Performance of delayed- enhancement magnetic resonance imaging with gadoversetamide contrast for the detection and assessment of myocardial infarction: an international, multicenter, double-blinded, randomized trial. Circulation. 2008;117:629-37.

2. Wagner A, Mahrholdt H, Holly TA, et al. Contrast enhanced MRI and routine single photon emission computed tomography (SPECT) perfusion imaging for detection of subendocardial myocardial infarcts: an imaging study. Lancet Lond Engl. 2003;361:374-9.

3. Arai AE, Schulz-Menger J, Berman D, et al. Gadobutrol-enhanced cardiac magnetic resonance imaging for detection of coronary artery disease. J Am Coll Cardiol. 2020;76:1536-47.

4. Mordi I, Bezerra H, Carrick D, Tzemos N. The combined incremental prognostic value of LVEF, late gadolinium enhancement, and global circumferential strain assessed by CMR. JACC Cardiovasc Imaging. 2015;8:540-9.

5. Dagres N, Hindricks G. Risk stratification after myocardial infarction: is left ventricular ejection fraction enough to prevent sudden cardiac death? Eur Heart J. 2013:34:1964-71.

6. Gavara J, Rodriguez-Palomares JF, Valente F, et al. Prognostic value of strain by tissue tracking cardiac magnetic resonance after ST-segment elevation myocardial infarction. JACC Cardiovasc Imaging. 2018:11:1448-57.

7. Eitel I, de Waha S, Wöhrle J, et al. Comprehensive prognosis assessment by CMR imaging after ST-segment elevation myocardial infarction. J Am Coll Cardiol. 2014:64:1217-26.

8. Lustig M, Donoho D, Pauly JM. Sparse MRI: the application of compressed sensing for rapid MR imaging. MagnReson Med. 2007:58:1182-95.

9. Kellman P, Larson AC, Hsu LY, et al. Motion-corrected free-breathing delayed enhancement imaging of myocardial infarction. MagnReson Med. 2005;53:194-200. 
10. Kido T, Kido T, Nakamura M, et al. Assessment of left ventricular function and mass on free-breathing compressed sensing real-time cine imaging. Circ J. 2017;81:1463-8.

11. Budjan J, Haubenreisser $H$, Henzler T, et al. Rapid functional cardiac imaging after gadolinium injection: evaluation of a highly accelerated sequence with sparse data sampling and iterative reconstruction. Sci Rep. 2016;6:38236

12. Sudarski $\mathrm{S}$, Henzler $\mathrm{T}$, Haubenreisser $\mathrm{H}$, et al. Free-breathing sparse sampling cine MR imaging with iterative reconstruction for the assessment of left ventricular function and mass at 3.0 T. Radiology. 2017;282:74-83.

13. Piehler KM, Wong TC, Puntil KS, et al. Free-breathing, motion-corrected late gadolinium enhancement is robust and extends risk stratification to vulnerable patients. Circ Cardiovasc Imaging. 2013;6:423-32.

14. Tonino PA, Fearon WF, De Bruyne B, et al. Angiographic versus functional severity of coronary artery stenoses in the FAME study fractional flow reserve versus angiography in multivessel evaluation. J Am Coll Cardiol. 2010;55:2816-21.

15. Menacho K, Ramirez S, Segura P, et al. INCA (Peru) study: impact of non-invasive cardiac magnetic resonance assessment in the developing world. J Am Heart Assoc. 2018;7:e008981.

16. Van Geuns RJ, Baks T, Gronenschild EH, et al. Automatic quantitative left ventricular analysis of cine MR images by using three-dimensional information for contour detection. Radiology. 2006;240:215-21.

17. Sievers B, Kirchberg S, Bakan A, Franken U, Trappe HJ. Impact of papillary muscles in ventricular volume and ejection fraction assessment by cardiovascular magnetic resonance. J Cardiovasc Magn Reson. 2004;6:9-16.

18. Mahrholdt H, Wagner A, Judd RM, Sechtem U, Kim RJ. Delayed enhancement cardiovascular magnetic resonance assessment of nonischaemic cardiomyopathies. Eur Heart J. 2005;26:1461-74.

19. Desroche LM, Milleron O, Safar B, et al. Cardiovascular magnetic resonance may avoid unnecessary coronary angiography in patients with unexplained left ventricular systolic dysfunction: a retrospective diagnostic pilot study. J Card Fail. 2020;26:1067-74.

20. Flett AS, Hasleton J, Cook C, et al. Evaluation of techniques for the quantification of myocardial scar of differing etiology using cardiac magnetic resonance. JACC Cardiovasc Imaging. 2011;4:150-6.

21. Bozkurt B, Coats AJS, Tsutsui $H$, et al. Universal definition and classification of heart failure: a report of the Heart Failure Society of America, Heart Failure Association of the European Society of Cardiology, Japanese Heart Failure Society and Writing Committee of the Universal Definition of Heart Failure: Endorsed by the Canadian Heart Failure Society, Heart Failure Association of India, Cardiac Society of Australia and New Zealand, and Chinese Heart Failure Association. Eur J Heart Fail. 2021;23:352-80.

22. Allen BD, Carr M, Botelho MP, et al. Highly accelerated cardiac MRI using iterative SENSE reconstruction: initial clinical experience. Int J CardiovasC Imaging. 2016:32:955-63.

23. Slomka PJ, Fieno D, Ramesh A, et al. Patient motion correction for multiplanar, multi-breath-hold cardiac cine MR imaging. J MagnReson Imaging. 2007;25:965-73.

24. Yang AC, Kretzler M, Sudarski S, et al. Sparse reconstruction techniques in magnetic resonance imaging: methods, applications, and challenges to clinical adoption. Invest Radiol. 2016;51:349-64.

25. Usman M, Atkinson D, Odille F, et al. Motion corrected compressed sensing for free-breathing dynamic cardiac MRI. Magn Reson Med. 2013;70:504-16.

26. Lin ACW, Strugnell W, Riley R, et al. Higher resolution cine imaging with compressed sensing for accelerated clinical left ventricular evaluation. J MagnReson Imaging. 2017:45:1693-9.

27. Ledesma-Carbayo MJ, Kellman P, Arai AE, McVeigh ER. Motion corrected free-breathing delayed-enhancement imaging of myocardial infarction using nonrigid registration. J Magn Reson Imaging. 2007;26:184-90.

28. Fan H, Li S, Lu M, et al. Myocardial late gadolinium enhancement: a head-to-head comparison of motion-corrected balanced steady-state free precession with segmented turbo fast low angle shot. Clin Radiol. 2018;73:593.e1-593.e9.

29. Vincenti G, Monney P, Chaptinel J, et al. Compressed sensing singlebreath-hold CMR for fast quantification of LV function, volumes, and mass. JACC Cardiovasc Imaging. 2014;7:882-92.
30. Kwong RY, Chan AK, Brown KA, et al. Impact of unrecognized myocardial scar detected by cardiac magnetic resonance imaging on event-free survival in patients presenting with signs or symptoms of coronary artery disease. Circulation. 2006;113:2733-43.

31. Lintingre PF, Nivet $H$, Clément GS, et al. High-resolution late gadolinium enhancement magnetic resonance for the diagnosis of myocardial infarction with nonobstructed coronary arteries. JACC CardiovasC Imaging. 2020;13:1135-48.

32. Savarese $G$, Stolfo D, Sinagra G, et al. Heart failure with mid-range or mildly reduced ejection fraction. Nat Rev Cardiol. 2021.

\section{Publisher's Note}

Springer Nature remains neutral with regard to jurisdictional claims in published maps and institutional affiliations.
Ready to submit your research? Choose BMC and benefit from:

- fast, convenient online submission

- thorough peer review by experienced researchers in your field

- rapid publication on acceptance

- support for research data, including large and complex data types

- gold Open Access which fosters wider collaboration and increased citations

- maximum visibility for your research: over $100 \mathrm{M}$ website views per year

At BMC, research is always in progress.

Learn more biomedcentral.com/submissions 